\title{
Quarkonia and Hybrids from the Lattice
}

\section{Michael}

Theoretical Physics Division,_Dept. Math. Sci., University of Liverpool, Liverpool L69 3BX, UK Email: 'c.michael@liv. ac.uki,

ABstract: The status of lattice determinations of quarkonia and hybrid meson spectra is presented and compared with experiment. Both quenched and unquenched results are discussed. Hybrid meson decays are considered.

$\mathrm{T}$ HIS PAPER discusses the consequences from first principles of QCD for the heavy quarkantiquark bound states: quarkonia and hybrid mesons. This is indeed the area of hadron spectroscopy where the underlying QCD structure is most readily discerned. The heavy quarks act as sources for the colour fields which provide the binding. It was realised early on [1] [1] that a combination of a short-range gluon exchange component and a long-range confining force is sufficient to give a good qualitative description of experimental data on quarkonia spectra. This can be made more precise by explicit lattice QCD evaluations.

Hybrid mesons are defined as those in which the gluonic component is non-trivial. The easiest way to ensure this is to require a spin-exotic state (where the $J^{P C}$ value cannot be attained from $Q \bar{Q}$ alone). For heavy quarks at separation $R$, the potential energy of these gluonic excitations can be established directly by lattice calculations [2]. This enables evaluation of the mass and properties of such hybrid mesons.

Here we summarise the various lattice approaches to heavy quark bound states and present the latest results. The most thorough lattice studies have been conducted in the quenched approximation. We discuss, however, evidence for differences between these lattice calculations in the quenched approximation and those with $N_{f}=$ 2 flavours of light sea quarks in the vacuum.

\section{Lattice QCD and heavy quarks}

The simplest way to treat a heavy quark on the lattice is to approximate it as static. The heavy quark propagators are then trivial to evaluate since they are products of time-directed gauge links. This enables potentials between such static colour sources at separation $R$ to be defined and the resulting spectrum of quarkonia can then be evaluated exactly from these potentials using the Schrödinger equation in the Born-Oppenheimer or adiabatic approximation. One advantage of this approach is that the continuum limit (lattice spacing $a \rightarrow 0$ ) can be readily taken. Moreover, lattice results at small separation in terms of the lattice spacing ( small $R / a$ ) can be corrected by hand for the lattice artifacts which arise since the lattice spatial symmetry is cubic rather than the continuum case which has the full rotation group.

In practice, however, the $b$ and $c$ quarks are not sufficiently heavy that this static approach is exact. Corrections can be arranged in powers of $1 / m_{Q}$ and can be evaluated in principle using the heavy quark effective theory (HQET). Examples of such calculations are the determination of the spin-orbit and spin-spin potentials between static quarks as well as velocity-dependent terms in the static potential itself.

To explore retardation effects, one needs a formalism in which the heavy quarks are moving. One promising approach is to expand the full theory as an effective lagrangian in powers of $v / c$ of the heavy quarks. This is the NRQCD scheme and the leading retardation effect in NRQCD 
comes from the p.A coupling between a quark colour charge in motion and the gluon field. Heavy quark propagators are relatively easy to evaluate in NRQCD since the heavy quarks do not propagate backwards in time. Because there are contributions in the effective lagrangian approach of the form $1 / m_{Q} a$, the continuum limit as $a \rightarrow 0$ is not to be taken: instead extra terms providing matching with the continuum to higher powers in $a$ and involving higher powers of $v / c$ in the effective lagrangian are needed to increase accuracy. The coefficients of these terms should ideally be determined non-perturbatively but in practice the lowest order perturbative expressions (tadpole improved) are usually used. This makes it difficult to estimate the systematic errors in the NRQCD approach. Note that corrections to the lattice cubic symmetry to restore rotational invariance will come from such higher order terms.

Without any approximation, the lattice formalism for relativistic quarks (Wilson-Dirac or staggered) can be applied directly to heavy quarks. Provided that $m_{Q} a<<1$, this approach is quite straightforward. Thus only $c$ quarks are tractable this way with present lattice spacings. This is a useful complement to the other two approaches which are less reliable for the case of the lighter $c$ quarks.

\section{Quarkonia}

In the static approximation, a potential $V(R)$ between static quarks in the fundamental representation of colour and at separation $R$ can be extracted from the lattice. In the quenched approximation, this evaluation goes back to the early 1980's. The salient features are a behaviour like $e / R$ at small $R$ and like $\sigma R$ at large $R$. Here $\sigma$ is the string tension and $e$ is related to the running coupling $\alpha_{s}$ (indeed this is one way to determine $\alpha_{s}$ from the lattice lattice potential (labelled $\Sigma_{g}^{+}$) and the wavefunctions and energy levels are illustrated in figure

The $b \bar{b}$ spectrum evaluated from this lattice potential in the Born-Oppenheimer approximation was found not to agree precisely with experiment. One way to quantify this is that the energy level ratio $\frac{1 P-1 S}{2 S-1 S}$ is around 0.71 from the quenched lattice $[3]]$ while it is 0.78 from exper- iment (for the spin averaged $\mathrm{S}$ and $\mathrm{P}$-wave $b \bar{b}$ states). For a more thorough discussion of this, including the effect of velocity dependent terms in the potential, see ref [5].

This can be understood as a consequence of the quenched approximation. The value of the Coulomb coefficient $e$ is expected in lowest order of perturbation theory to contain a factor $\left(33-2 N_{f}\right)^{-1}$ and so will increase as sea quarks (with $N_{f}=2$, say) are included. This effect has been confirmed by explicit lattice calculation in-

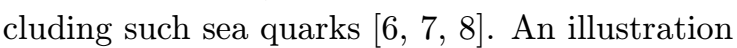
is shown in figure $\overline{2}$. Indeed effects of including sea quarks seem to be comparable to those expected from perturbation theory although much work still needs to be done to include even lighter sea quarks in the vacuum so that the extrapolation to light sea quarks is under better control. The consequence of this increase in the depth of the potential at small $R$ is that the $1 \mathrm{~S}$ level will be moved down in energy, resulting in an increase of the ratio $\frac{1 P-1 S}{2 S-1 S}$ to bring it more into line with experiment. For this reason we will use differences with the $2 \mathrm{~S}$ energy to estimate the hybrid energy levels subsequently from quenched calculations. Note that, experimentally, the $1 P-1 S$ energy splitting is very similar for $c \bar{c}$ and $b \bar{b}$. This coincidence has been used as evidence that this quantity is insensitive to quark masses and hence a good point of comparison between lattice calculations and experiment. While this may be true for valence quarks, it not likely to be valid for sea quarks, since, as we have discussed above, the $1 S$ level is especially sensitive to the sea quark effects.

The other régime in which sea quarks will make a definite impact is in the large $R$ region. It will become energetically favourable to create two heavy-light mesons $(Q \bar{q})$ of energy $2 m_{Q \bar{q}}$ when this energy is less than $V(R)$. This phenomenon is known as string breaking since as $R$ is increased the colour flux between the static sources breaks with the formation of a light quarkantiquark pair. From the lattice mass values of the heavy-light mesons, this string breaking can be predicted to occur at around $1.2 \mathrm{fm}$ - an illustration is shown in figure $\bar{\beta}_{r}^{\prime}$ Lattice studies of the potential $V(R)$ using generalised Wilson loops have not reached sufficient precision to ob- 


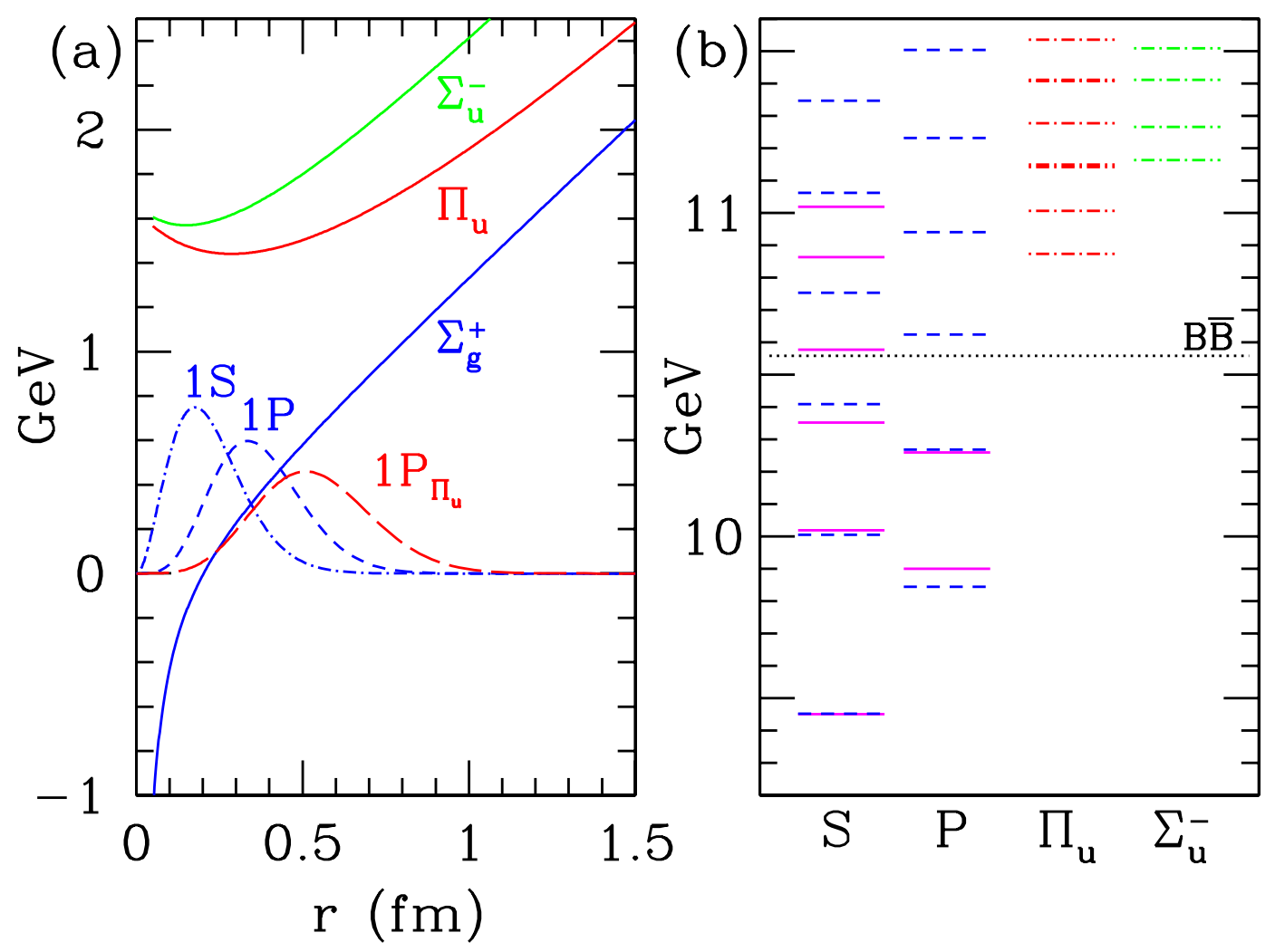

Figure 1: (a)Potentials and wave functions for $b \bar{b}$ hadrons in the Born-Oppenheimer approximation from ref [10] for quenched QCD. (b)The resulting $S$ and $P$ spin-averaged energy levels in the ground state potential (labelled $\Sigma_{g}^{+}$) are compared with experiment(shown as solid lines). The energies of some of the lower lying hybrid excitations are also illustrated, for example in the $\Pi_{u}$ potential as will be discussed in section 3 .

serve this directly. It is known from studies of the adjoint potential [122] that a variational approach involving both the string states and the meson-antimeson states will be needed to obtain accurate energy estimates at these large separations - this is under way [1] 3

NRQCD calculations of quarkonia show very similar results to the potential approach described above. A comparison [1] of the NRQCD result with the potential approach shows no significant evidence for retardation effects. Indeed differences among NRQCD results i10i] arising from different lattice spacings and different treatment of higher order corrections are of the same magnitude as their difference from the

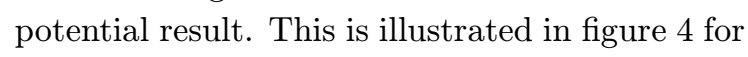
the quarkonium $1 P$ and $2 S$ excitations.

A quenched lattice study of quarkonia using relativistic quarks [i] 1 il] also shows similar results to those found by the other methods described above.

\subsection{Quarkonia decays}

A very approximate study of some quarkonium decays can be made in the Born-Oppenheimer approximation using esentially the methods of atomic physics: overlaps of wave functions. In particular the decay to lepton pairs will be governed by the wave function at the origin. In practice the corrections to the non-relativistic approach for decays are much larger than for the energy values, so this approach is rather imprecise.

Hadronic decays (such as $\Upsilon(4 S) \rightarrow B \bar{B}$ ) are of interest because they proceed by string breaking: a light quark pair is created which then results in a pair of heavy-light mesons being produced. This process is accessible in principle from lattice calculations [1] 

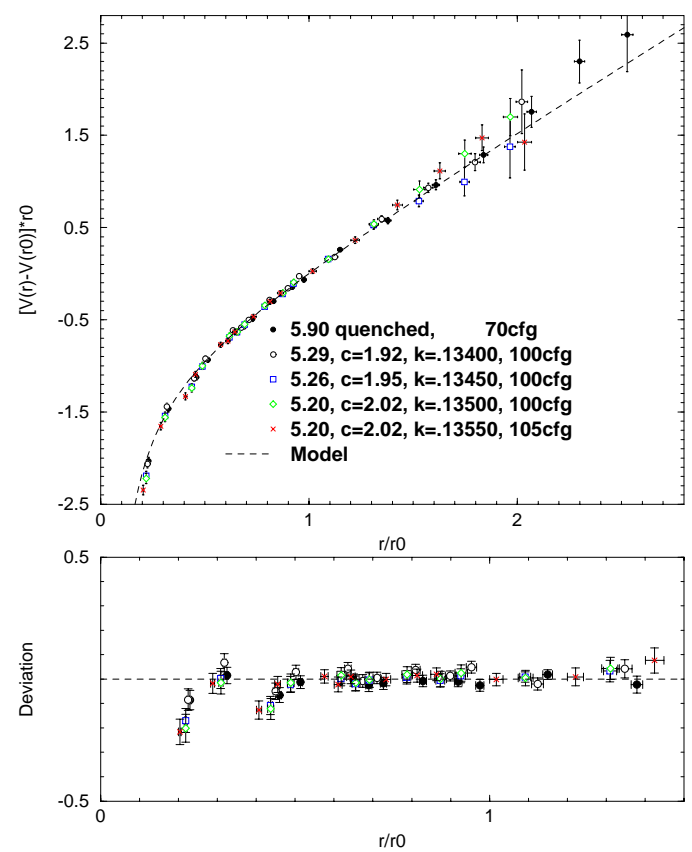

Figure 2: The potential $V(R)$ between static quarks with $N_{f}=0$ and 2 flavours of sea quark from ref in units of $r_{0} \approx 0.5 \mathrm{fm}$. The lower part shows the differences from the common dotted curve which emphasises the increase in the strength of the $e / R$ term as the sea quark mass is decreased (larger $\kappa$ value).

from the splitting of the energy levels caused by string breaking, one can estimate the decay rate [in

\section{Hybrid Mesons}

The static quark approach gives a very straightforward way to explore hybrid quarkonia. These will be $Q \bar{Q}$ states in which the gluonic contribution is excited. The ground state of the gluonic degrees of freedom has been explored on the lattice, and, as expected, corresponds to a symmetric cigar-like distribution of colour flux between the two heavy quarks. One can then construct less symmetric colour distributions which would correspond to gluonic excitations.

The way to organise this is to classify the gluonic fields according to the symmetries of the system. This discussion is very similar to the description of electron wave functions in diatomic molecules. The symmetries are (i) rotation around the separation axis $z$ with representations labelled by $J_{z}$ (ii) CP with representations labelled by $g$

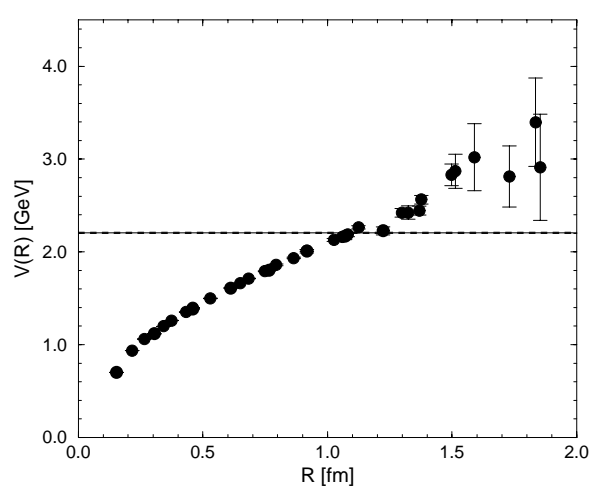

Figure 3: The potential $V(R)$ between static quarks with $N_{f}=2$ flavours of sea quark from ref $[7, n$ and the energy corresponding to twice the ground state heavy-light meson mass. This shows that the string breaking region, where they cross, is at $R \approx 1.2 \mathrm{fm}$. No sign of string breaking is seen in this figure because the Wilson loop operators used have a very small overlap with the meson-meson configuration.

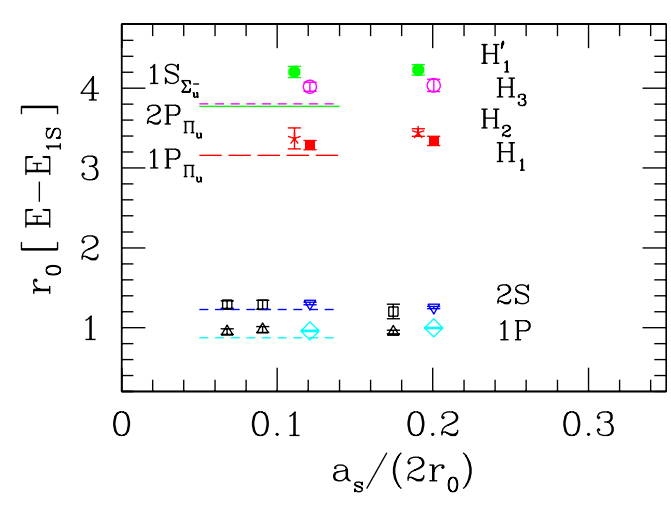

Figure 4: Excitation energies above the ground state for $b \bar{b}$ states from the potential approach (horizontal lines) and NRQCD (symbols) at the spatial lattice spacing shown in units of $2 r_{0} \approx 1 \mathrm{fm}$ from [i10] for quenched lattices. The NRQCD hybrid operators $H_{1}$ and $H_{2}$ correspond to the $L^{P C}=1^{+-}$and $1^{-+}$ excitations respectively - these excitations are degenerate in the potential approach.

and $u$ and (iii) $\mathrm{C} \mathcal{R}$. Here $\mathrm{C}$ interchanges $Q$ and $\bar{Q}, \mathrm{P}$ is parity and $\mathcal{R}$ is a rotation of $180^{\circ}$ about the mid-point around the $y$ axis. The $\mathrm{C} \mathcal{R}$ opera- 


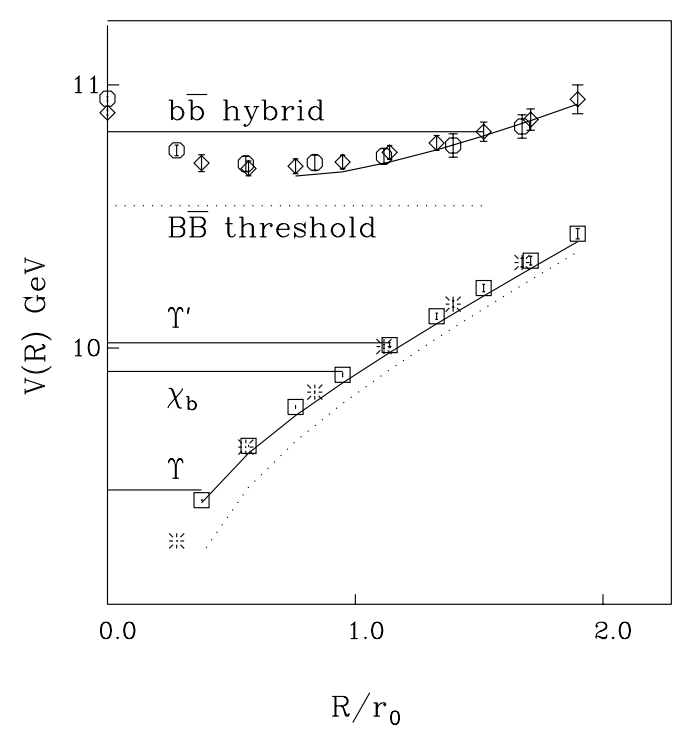

Figure 5: An illustration of the $b \bar{b}$ states in the static potential and in the first gluonic excitation from ref[[3] using quenched lattices. Here $2 r_{0} \approx 1$ $\mathrm{fm}$. The dotted curve illustrates the potential that would be needed to reproduce more closely the observed spectrum of $1 S, 1 P$ and $2 S$ states.

tion is only relevant to classify states with $J_{z}=0$. The convention is to label states of $J_{z}=0,1,2$ by $\Sigma, \Pi, \Delta$ respectively.

In lattice studies the rotation around the separation axis is replaced by a four-fold discrete symmetry and states are labelled by representations of the discrete group $D_{4 h}$. The ground state configuration of the colour flux is then $\Sigma_{g}^{+}\left(A_{1 g}\right.$ on the lattice). The exploration of the energy levels of other representations has a long history in lattice studies [2] found to be the $\Pi_{u}\left(E_{u}\right.$ on a lattice $)$ - see figure $\overline{1}$, for an illustration. This can be visualised as the symmetry of a string bowed out in the $x$ direction minus the same deflection in the $-x$ direction (plus another component of the twodimensional representation with the transverse direction $x$ replaced by $y$ ), corresponding to flux states from a lattice operator which is the difference of U-shaped paths from quark to antiquark of the form $\sqcap-\sqcup$.

Recent lattice studies [1 1001$]$ have used an asymmetric space/time spacing which enables excited states to be determined in a well controlled way. Results are shown in figure $\overline{6}_{\mathbf{G}}^{\mathbf{6}}$ for a large variety

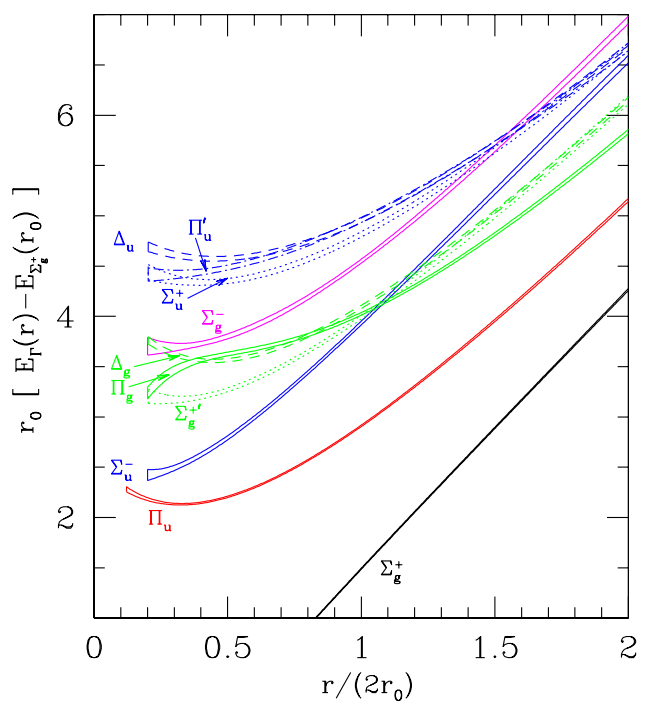

Figure 6: The potential energies, extrapolated to the continuum limit, for different gluonic excitations from ref[']10'] for quenched lattices. Here $2 r_{0} \approx 1 \mathrm{fm}$.

of gluonic excitations. These results confirm the finding that the $\Pi_{u}$ excitation is the lowest lying and hence of most relevance to spectroscopy.

From the potential corresponding to these excited gluonic states, one can determine the spectrum of hybrid quarkonia using the Schrödinger equation in the Born-Oppenheimer approximation. This approximation will be good if the heavy quarks move very little in the time it takes for the potential between them to become established. More quantitatively, we require that the potential energy of gluonic excitation is much larger than the typical energy of orbital or radial

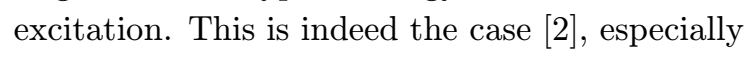
for $b$ quarks. Another nice feature of this approach is that the self energy of the static sources cancels in the energy difference between this hybrid state and the $Q \bar{Q}$ states. Thus the lattice approach gives directly the excitation energy of each gluonic excitation.

The $\Pi_{u}$ symmetry state corresponds to excitations of the gluonic field in quarkonium called magnetic (with $L^{P C}=1^{+-}$) and pseudo-electric (with $1^{-+}$) in contrast to the usual P-wave orbital excitation which has $L^{P C}=1^{--}$. Thus we expect different quantum number assignments from those of the gluonic ground state. Indeed combining with the heavy quark spins, we get a 
degenerate set of 8 states with $J^{P C}=1^{--}, 0^{-+}$, $1^{-+}, 2^{-+}$and $1^{++}, 0^{+-}, 1^{+-}, 2^{+-}$respectively. Note that of these, $J^{P C}=1^{-+}, 0^{+-}$and $2^{+-}$ are spin-exotic and hence will not mix with $Q \bar{Q}$ states. They thus form a very attractive goal for experimental searches for hybrid mesons. Illustrations of the spectrum of such spin-exotic hybrid mesons are given in figure $\overline{1} 1$, and $\overline{15}$.

The eightfold degeneracy of the static approach will be broken by various corrections. As an example, one of the eight degenerate hybrid states is a pseudoscalar with the heavy quarks in a spin triplet. This has the same overall quantum numbers as the S-wave $Q \bar{Q}$ state $\left(\eta_{b}\right)$ which, however, has the heavy quarks in a spin singlet. So any mixing between these states must be mediated by spin dependent interactions. These spin dependent interactions will be smaller for heavier quarks. It is of interest to establish the strength of these effects for $b$ and $c$ quarks. Another topic of interest is the splitting between the spin exotic hybrids which will come from the different energies of the magnetic and pseudo-electric gluonic excitations.

One way to study this is using the NRQCD approach which enables the $L^{P C}=1^{+-}$and $1^{-+}$excitations to be separated in a spin averaged approach. Lattice results [1] $\left.\overline{0}_{1}^{\prime}\right]$ indicate no statistically significant splitting (see figure although the $1^{+-}$excitation does lie a little lighter. This would imply, after adding in heavy quark spin, that the $J^{P C}=1^{-+}$hybrid was the lightest spin exotic. In principle the NRQCD approach, by adding spin-dependent terms in the Lagrangian, can address the full splitting of the 8 levels. However, as we shall also discuss in connection with propagating quark approaches, the mixing of non spin exotic states with $Q \bar{Q}$ may confuse this situation. Including spin-dependent terms in a NRQCD study of hybrids does give a relatively large spin splitting among the triplet states. Unfortunately this study has only considered magnetic gluonic excitations so cannot address the splitting between spin exotic hybrids.

Confirmation of the ordering of the spin exotic states also comes from lattice studies with

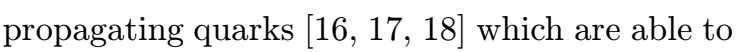
measure masses for all 8 states. We discuss this evidence in more detail below - see also figure
Within the quenched approximation, the lattice evidence for $b \bar{b}$ quarks points to a lightest hybrid spin exotic with $J^{P C}=1^{-+}$at an energy given by $\left(m_{H}-m_{2 S}\right) r_{0}=1.8$ (static poten-

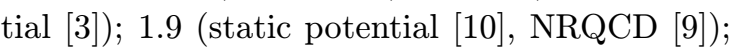
2.0 (NRQCD [10 $\left.0_{1}^{\prime \prime}\right)$. These results can be summarised as

$$
\left(m_{H}-m_{2 S}\right) r_{0}=1.9 \pm 0.1
$$

Here $r_{0}$ is defined implicitly by the static force as $r^{2} F(r)=1.65$ at $r=r_{0}$ and is a well measured quantity on a lattice derived from the static potential $V(R)$ at $r \approx 0.5 \mathrm{fm}$. Within the quenched approximation, where different experimental observables differ by of order $10 \%$, the overall scale is uncertain but we choose $r_{0}^{-1}=390 \mathrm{MeV} \pm$ $10 \%$. Using the experimental mass of the $\Upsilon(2 S)$, this implies that the lightest spin exotic hybrid is at $m_{H}=10.76(7) \mathrm{GeV}$. Above this energy there will be many more hybrid states, many of which will be spin exotic.

Some preliminary lattice studies have been made including sea quarks. As yet only sea quark masses down to the strange quark mass have been explored and hence the extrapolation to realistic sea quark masses is not yet well established. Indeed, pushing to lower sea quark masses is the main remaining challenge in lattice gauge theory. The light propagating quark case has been explored [1] 1 '] but no significant differences are found from using quenched vacua. The excited gluonic static potential has also been determined including sea quarks ( $N_{f}=2$ flavours) and no significant difference is seen [1 $\left.19 \overline{9}_{1}^{\prime}\right]$. Thus the quenched estimates given above are not superseded. Note, however, that hybrid states can mix with $Q \bar{Q} q \bar{q}$ states - for instance with their decay products as we shall discuss in the next section. This mixing is, in principle, enabled in a lattice study with sea quarks.

\subsection{Light quark hybrid mesons}

Here we focus on lattice results for hybrid mesons made from light quarks using fully relativistic propagating quarks. There will be no mixing with $q \bar{q}$ mesons for spin-exotic hybrid mesons and these are of special interest. The first study of this area was by the UKQCD Collaboration [1 16 ] 


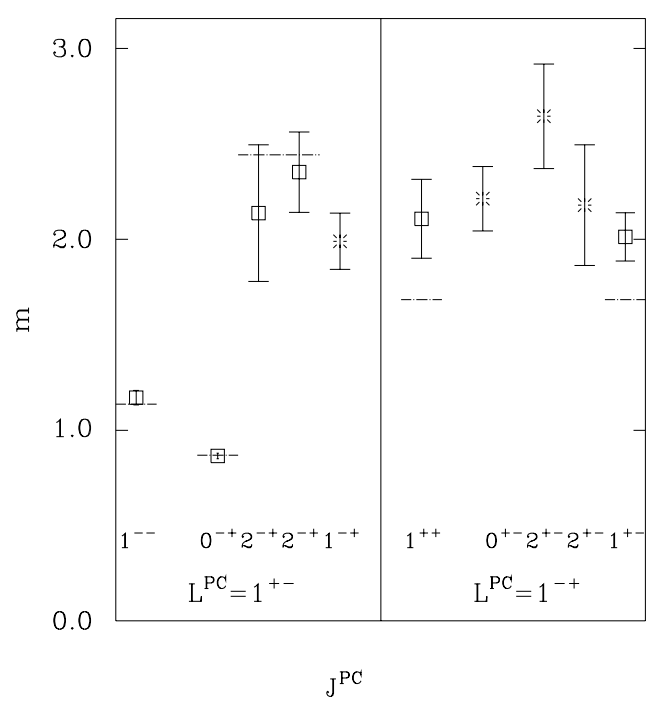

Figure 7: The masses in $\mathrm{GeV}$ of states of $J^{P C}$ built from hybrid operators with strange quarks, spinexotic $(*)$ and non-exotic (squares). The dot-dashed lines are the mass values found for $s \bar{s}$ operators. Quenched lattice results from ref[1] 16

who used operators motivated by the heavy quark studies referred to above. Using non-local operators, they studied all $8 J^{P C}$ values coming from $L^{P C}=1^{+-}$and $1^{-+}$excitations. The resulting mass spectrum is shown in figure $\overline{\overline{7}}$, where the $J^{P C}=1^{-+}$state is seen to be the lightest spin-exotic state with a statistical significance of 1 standard deviation. The statistical error on the mass of this lightest spin-exotic meson is $7 \%$ but, to take account of systematic errors from the lattice determination, a mass of 2000(200) $\mathrm{MeV}$ is quoted for this hybrid meson with $s \bar{s}$ light quarks. Although not directly measured, the corresponding light quark hybrid meson would be expected to be around $120 \mathrm{MeV}$ lighter.

One feature clearly seen in figure $\overline{\overline{7}}$ is that non spin-exotic mesons created by hybrid meson operators have masses which are very similar to those found when the states are created by $q \bar{q}$ operators. This suggests that there is quite strong coupling between hybrid and $q \bar{q}$ mesons even in the quenched approximation. This would imply that the $\pi(1800)$ is unlikely to be a pure hybrid, for example.

A second lattice group has also evaluated hybrid meson spectra with propagating quarks from

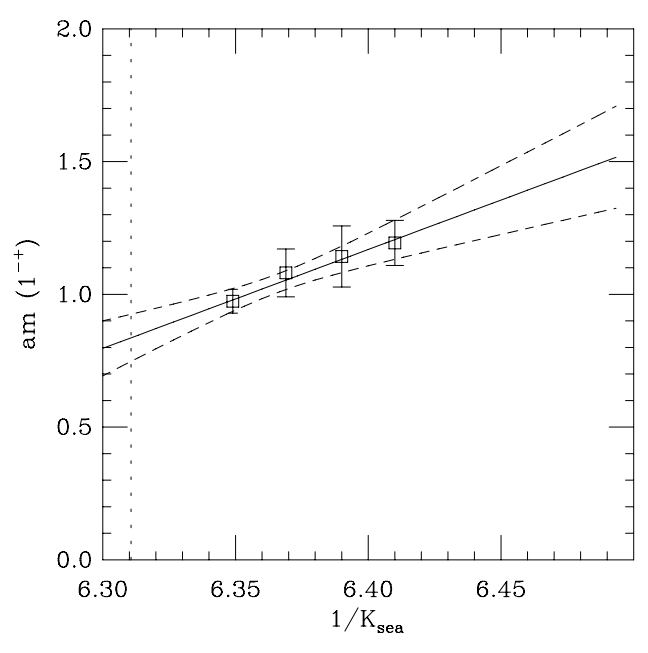

Figure 8: The extrapolation in sea quark mass (in lattice units) from ref [18 for the $1^{-+}$hybrid meson. The dotted vertical line corresponds to light sea quarks.

quenched lattices. They obtain [1] $\left.\overline{7}_{1}\right]$ masses of the $1^{-+}$state with statistical and various systematic errors of 1970(90)(300) MeV, 2170(80)(100)(100) $\mathrm{MeV}$ and 4390(80)(200) $\mathrm{MeV}$ for $n \bar{n}, s \bar{s}$ and $c \bar{c}$ quarks respectively. For the $0^{+-}$spin-exotic state they have a noisier signal but evidence that it is heavier. They also explore mixing matrix elements between spin-exotic hybrid states and 4 quark operators.

Recently a first attempt has been made [1] 1 ['] to determine the hybrid meson spectrum using full QCD. The sea quarks used have several different masses and an extrapolation is made to the limit of physical sea quark masses, yielding a mass of 1.9(2) GeV for the lightest spin-exotic hybrid meson, which they again find to be the $1^{-+}$. In principle this calculation should take account of sea quark effects such as the mixing between such a hybrid meson and $q \bar{q} q \bar{q}$ states such as $\eta \pi$. As illustrated in figure $\overline{1}_{1}^{\prime}$, the calculations are performed for quite heavy sea quarks (the lightest being approximately the strange quark mass) and then a linear extrapolation is made. It is quite possible, however, that such mixing effects turn on non-linearly as the sea quark masses are reduced. The systematic error from this possibility is difficult to quantify.

The three independent lattice calculations of 
the light hybrid spectrum are in good agreement with each other. They imply that the natural energy range for spin-exotic hybrid mesons is around $1.9 \mathrm{GeV}$. The $J^{P C}=1^{-+}$state is found to be lightest. It is not easy to reconcile these lattice results with experimental indications [201] for resonances at $1.4 \mathrm{GeV}$ and $1.6 \mathrm{GeV}$, especially the lower mass value. Mixing with $q \bar{q} q \bar{q}$ states such as $\eta \pi$ is not included for realistic quark masses in the lattice calculations. This can be interpreted, dependent on one's viewpoint, as either that the lattice calculations are incomplete or as an indication that the experimental states may have an important meson-meson component in them.

\subsection{Hybrid meson decays}

One clear feature of heavy quark hybrid mesons is that they have very extended wavefunctions since the potential that binds them is relatively flat. This has implications for their production and decay. For instance, any vector state will only be weakly produced in $e^{+} e^{-}$collisions because the wave function at the origin will be small.

Given our mass estimates above, the open channels for decay of a $J^{P C}=1^{-+}$hybrid include $B \bar{B}, B \bar{B}^{*}, \eta_{b} \eta, \eta_{b} \eta^{\prime}, \Upsilon(1 S) \omega$ and $\Upsilon(1 S) \phi$. Selection rules have been proposed for hybrid decays, for example [21 $\overline{1}_{1}^{\prime}$ that $H \nrightarrow X+Y$ if $X$ and $Y$ have the same non-relativistic structure and each has $L=0$. This would rule out $B \bar{B}$ and $B \bar{B}^{*}$ and the analogous cases for charm quarks.

This selection rule can be addressed directly from the static quark approach. The symmetries in this case of rotations about the separation axis, etc have to be preserved in the strong decay. From the initial state with the gluonic field in a given symmetry representation, the $q \bar{q}$ pair must be produced in the decay in such a way that the combined symmetry of the quark pair and the final gluonic distribution matches the initial representation.

For the ground state of the gluonic excitation (non-hybrid) we have $J_{z}=0$ and even $C P$. Thus, for this state to decay to $(Q \bar{q})(\bar{Q} q)$ with each heavy-light meson having $L=0$, the final gluonic distribution is also symmetric (actually it is essentially two spherical blobs around each static source binding the heavy light mesons).
Then any $q \bar{q}$ pair production has to respect this symmetry and have $J_{z}=0$ and even $C P$. Since there is no orbital angular momentum, the $C P$ condition then requires $S_{q \bar{q}}=1$, a triplet state. This is just a derivation of what is called the ${ }^{3} P_{0}$ model of decays: the light quark-antiquark is produced in a triplet state. This spin assignment can be tested by the ratio of $B \bar{B}, B \bar{B}^{*}$ and $B^{*} \bar{B}^{*}$ decays.

For the $J^{P C}=1^{-+}$hybrid we have a gluonic field with $J_{z}=1$ and odd $C P$. For the case of decay to a $(Q \bar{q})(\bar{Q} q)$ with each heavy-light meson having $L=0$, this would imply that the $q \bar{q}$ would have to be produced with $J_{z}=1$ and odd $C P$. This is not possible since the triplet state would have even $C P$ while the singlet state cannot have $J_{z}=1$. This is then equivalent to the selection rule described above. There will presumably be small corrections to this selection rule coming from retardation effects. Decay to $(Q \bar{q})(\bar{Q} q)$ with one heavy-light meson having a non-zero orbital excitation is allowed from symmetry but is not allowed energetically with conventional mass assignments for the $\mathrm{P}$-wave excited B meson multiplet.

Decays to $(Q \bar{Q})(q \bar{q})$ are also possible since there is enough excitation energy to create a light quark meson. This meson must be created in a flavour singlet state and the lightest candidates are $\eta$ and $\omega$. In a lattice context, this production is via a disconnected quark loop with $s, u$ and $d$ quark contributions of similar strength. The flavour singlet mixture of $\eta$ and $\eta^{\prime}$ (mainly $\eta^{\prime}$ ) and the singlet mixture of the vector mesons (which includes a substantial $\omega$ component) are expected to be coupled most strongly.

So allowed decays are $\eta_{b} \eta, \eta_{b} \eta^{\prime}, \Upsilon(1 S) \omega$ and $\Upsilon(1 S) \phi$. Here the light meson must have $J_{z}=1$ and together with the $C P$ constraint, this implies that the light meson must be in a $P$-wave with respect to the heavy quark meson.

$S$-wave decays to $\eta_{b} f_{1}$ and $\Upsilon(1 S) h$ are also allowed although there may be insufficient phase space. (Here the $f_{1}$ and $h$ are $J^{P C}=1^{++}$and $1^{-+}$flavour singlet mesons).

As for the case of quarkonium decays, it is possible in principle to explore on the lattice some aspects of these decays. One can study matrix elements between ground states which are degen- 
erate in energy such as the $1^{-+}$hybrid and the $\eta_{b} \eta$ final state where the light quark mass is adjusted so that there is equal energy in both systems. This and similar lattice studies will enable some further guidance to be given for experimental searches for hybrid mesons.

\section{Summary and Outlook}

One of the advantages of lattice studies is that, by varying the parameters such as quark masses, they can serve as very useful data to develop phenomenological models. One example is that the excitation spectrum of the potential between static quarks can be used to test QCD string excitation models. This has been much discussed for a review see ref [20 $2 \overline{2}]$.

At present, lattice studies are restricted to sea quark masses no no lighter than th strange quarkss. '- The_results _with such _sea _quarks show _rather modest changes from the quenched results as the sea quarks are included but this may change nonlinearly as the sea quark masses are further reduced. Thus the systematic error associated with the extrapolation in sea quark mass is very hard to estimate. The only way to circumscribe this systematic error is by evaluating explicitly with lighter sea quarks and this requirement is the remaining big computational challenge in the lattice approach.

Present lattice results for quarkonia are in quantitative agreement with experiment, taking into account the uncertainty in the extrapolation in sea quark mass.

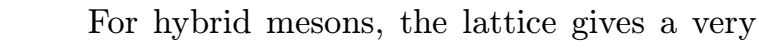

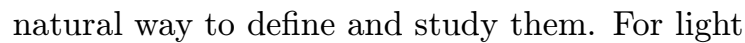
quark hybrids one can explore the spectrum for all $J^{P C}$ values, finding $\left[\overline{1} \overline{6}_{1}^{\prime}, \overline{1} \overline{7}_{1}, \overline{1} \overline{1} \bar{l}_{1}^{\prime}\right.$ a lightest spin-exotic hybrid with $J^{P C}=1^{-+}$and mass $1.9(2) \mathrm{GeV}$. This mass is significantly higher than mass values found [20 $\left.{ }^{\prime}\right]$ experimentally (1.4 and 1.6 GeV).

The situation for $c \bar{c}$ hybrid states is that neither the heavy quark lattice methods (potentials, NRQCD) nor the light quark methods (propagating quarks) are at their best in this quark mass region. Estimates $[\overline{3}, \overline{1} \overline{1}, \bar{p}$, for the lightest $c \bar{c}$ hybrid have been given from all three lattice meth- ods and lie around $\frac{H-1 S}{1 P-1 S} \approx 3.0$ but the systematic errors are quite large.

The situation is much better controlled for $b \bar{b}$ hybrids. We expect the lightest $b \bar{b}$ hybrid to have $J^{P C}=1^{-+}$and mass $10.75 \pm 0.10 \mathrm{GeV}$. It will be difficult to isolate such states experimentally but well worth the effort. The most likely decay modes of this hybrid meson are to a $b \bar{b}$ ground state meson $\left(\eta_{b}\right.$ or $\left.\Upsilon(1 S)\right)$ with the emission of a flavour singlet light quark meson $\left(\eta, \eta^{\prime}, \omega\right.$ or $\phi)$. Future lattice calculations should be able to study these and other decays.

\section{References}

[1] E. Eichten et al., 'Phys. Rev. D 21

[2] L.A. Griffiths, C. Michael and P.E.L. Rakow, Phys. Lett. B 129 (1983) 351,

[3] S. Perantonis and C. Michael 'Nucl. Phys. B' $\mathbf{3 4 7}(1990) 854$

[4] C. Michael, 'Phys. Lett. B $\mathbf{2} 83$ (1992) 103

[5] G. Bali and P. Boyle, hep-lat/98090180.

[6] SESAM Collaboration, U. Glässner et al., Phys. Lett. B $\mathbf{3 8 3}(1966) 98 ;$ S. Güsken, Nucl. Phys. (Proc. Suppl.)B 63 (1998) 16

[7] CP-PACS Collaboration, S. Aoki et al., Nucl. Phys. (Proc. Suppl.) $\mathbf{7 3}$ (1999) 216

[8] UKQCD Collaboration, J. Garden, Nucl. Phys. (Proc. Suppl.) (in press), hep-lat/9909066

[9] CP-PACS Collaboration, T. Manke et al., Phys. Rev. Lett. - 82 _ (1999) 4396; heplat/9909038; hep-lat/9909133

[10] K. Juge , J. Kuti and C. Morningstar, Phys.' Rev. Lett. $\mathbf{8 2}(1999)-4400 ;$, hep-lat/9909165

[11] P. Boyle (UKQCD Collaboration), heplat/9903017.

[12] C. Michael, Nucl. Phys. (Proc. Suppl.) B 26 (1992) 417

[13] P. Pennanen, C. Michael and A.M. Green (UKQCD Collaboration), Nucl. Phys. (Proc. Suppl.) (in press), hep-lat/9908032, and in preparation.

[14] C. DeTar, U. Heller and P. Lacock, Nucl. Phys. (Proc. Suppl.) (in press), hep-lat/9909078

[15] I. T. Drummond and R. R. Horgan, iPhys. Lett.' B $447(1999) 298$ 
[16] UKQCD Collaboration, P. Lacock et al.,Phys.' Rev. D 54 (1996) 6997', Phys. Lett. B 401' _ _ _ (1997)_308'

[17] C. Bernard et al., í

[18] P. Lacock and K. Schilling, Nucl. Phys. (Proc. Suppl.) B 73 (1999) 261, hep-lat/9809022

[19] G. Bali, hep-lat/9901023

[20] D. Thompson et al., PPhys. Rev. Lett. $\mathbf{7 9}(1997)$, $I^{-1630}$ S. U. Chung et al., Phys. Rev. D $\mathbf{6 0}$ (1999) 092001; D. Adams et al., iPhys. Rev. Lett. 81 (1998) 5760

[21] P. Page, 'Phys. Lett. B 402 (1997) 183'

[22] C. Michael, Proceedings of Confinement III, Newport News, hep-ph/9809211 